\title{
Influencia de los factores de localización en la fijación de los precios de los carburantes de automoción en España*
}

\author{
Alejandro Bello Pintado \\ Ignacio Contín-Pilart \\ Dpto. Gestión de Empresas \\ Universidad Pública de Navarra
}

\section{Resumen}

Este trabajo analiza el efecto de los factores locales de oferta en la fijación de los precios de los carburantes de automoción a nivel de estaciones de servicio en España. Para ello contamos con información sobre los precios que fijan, y sobre las características de los mercados locales en donde operan, 529 estaciones de servicio que representa fielmente la estructura competitiva del mercado español a nivel nacional y regional a lo largo de 2007. Nuestros resultados sugieren la existencia de imperfecciones en la competencia y muestran cómo la existencia de estaciones de servicio independientes en los mercados locales la intensifican.

Palabras Clave: Gasolinas, gasóleos, competencia, factores de localización.

Clasificación JEL: D40, D43, L11, L71, L81.

\begin{abstract}
This paper analyzes the effects of supply local factors on fuel automotive (gasoline and diesel) pricing behavior in Spain. In doing so, we have collected information about local market conditions in which operated a sample of 529 Spanish service stations in 2007. We have also gathered weekly prices set by such service stations over 2007. This sample of stations represents accurately the competitive structure, both at regional and national level, of the Spanish market for automotive fuels. Our results suggest that competition works "imperfectly" and show how the presence of independent service stations in local markets enhances competition.
\end{abstract}

Keywords: Gasoline, diesel, competition, supply local factors.

JEL Classification: D40, D43, L11, L71, L81.

\section{Introducción}

El contar con un sector de distribución de carburantes de automoción (gasolinas y gasóleos) eficiente resulta clave para el funcionamiento de la economía de cualquier país desarrollado. Tanto la gasolina como el gasóleo de automoción son inputs

* Los autores de este trabajo desean agradecer la financiación del Ministerio de Educación (SEC2007-67737-C03-02 y SEJ2007-67895-C04-01). 
básicos que hacen posible el transporte y desplazamiento diario de personas y mercancías por carretera ${ }^{1}$. Por lo tanto lograr que este sector opere bajo condiciones competitivas, lo que impulsaría ceteris paribus las ganancias de eficiencia, es un objetivo básico de los poderes políticos y de las autoridades regulatorias y de la competencia de cualquier país.

Como consecuencia del proceso de reestructuración y liberalización del sector petrolero que se llevó a cabo a lo largo de los años 80 y primeros 90 del siglo pasado, la industria española del petróleo se caracteriza, dentro del contexto europeo, por su elevada concentración industrial (García de Coca, 2004)². Las plantas de refino en España están en manos de sólo tres operadores (Repsol-Ypf, Cepsa-Total y BP), que a su vez mantienen una importante presencia en el capital de la Compañía Logística de Hidrocarburos (CLH). Esta empresa es considerada la "essential facility" del sector petrolero español, al ser la dueña de la red de oleoductos y de la mayor parte de la capacidad de almacenamiento de productos petrolíferos que no está en manos de las refinerías (Contín et al., 2001).

Asimismo, los tres empresas refineras tienen hoy en día una cuota de participación conjunta en la parte final del sector petrolero, la comercialización de carburantes de automoción a través de estaciones de servicio, de aproximadamente el setenta y cuatro por ciento; si bien, la concentración en esta fase ha ido disminuyendo paulatinamente desde la abolición del Monopolio en enero de 1993, como consecuencia de la entrada en el mercado español de un número importante de operadores internacionales, estaciones independientes y, en menor medida, de hipermercados.

El hecho de que los tres principales operadores con capacidad de refino tengan un peso tan destacado en la parte final del negocio petrolero, en particular RepsolYpf, y la elevada sensibilidad de las familias a las variaciones del precio de los carburantes de automoción, han generado desde prácticamente la misma abolición del Monopolio un debate recurrente, especialmente en épocas de fuertes aumentos de los precios spot y de los precios finales, sobre el grado de competencia y por lo tanto sobre el nivel de eficiencia alcanzado en este mercado.

Así, por ejemplo, la Comisión Nacional de la Competencia (CNC) en su último informe sobre este sector afirma: "la existencia de elementos tanto estructurales (elevada concentración) como de comportamiento estarían provocando la falta de competencia en el sector" (CNC, 2009; pag. 1). Señala como uno de los indicadores más importante de esa esta falta de competencia que la horquilla de precios antes de im-

${ }^{1}$ En cuanto al peso directo que tiene en las economías domésticas la factura derivada del uso de carburantes del automoción, la Encuesta de Presupuestos Familiares del INE señala que el gasto en “carburantes y lubricantes" representó en España el 4,29\% del gasto doméstico en 2007, lo que significó $1.372 €$ por familia ese año (CNC, 2009).

${ }^{2}$ La liberalización culminó con la abolición del Monopolio de Petróleos en enero de 1993. No obstante, y dada la elevada concentración del mercado, la fijación administrativa de precios fue sustituida por una regulación de precios máximos en junio de 1990. Estuvo vigente hasta junio de 1996 para el gasóleo de automoción y hasta septiembre de 1998 para la gasolina sin plomo de 95 octanos. 
puestos, tanto en el caso de la gasolina como en el del gasóleo, es "muy reducida"; situándose el precio medio en ambos casos cerca de su máximo3.

A lo largo de los últimos años, distintos trabajos académicos, citados más adelante, han analizado diversas cuestiones sobre el funcionamiento del sector petrolero español. No obstante, la mayoría de estos trabajos utilizan en sus análisis datos agregados y no analizan el impacto que los factores locales tienen en la fijación de los precios de las estaciones de servicio. Varios autores (Netz y Taylor, 2002; Lewis, 2008; Pennerstorfer, 2009) señalan que estos aspectos son fundamentales a la hora de entender el funcionamiento competitivo del sector de la distribución de carburantes de automoción. En este misma línea, la CNC (2009) señala que la diferenciación espacial asociada a la localización geográfica concreta de cada estación de servicio es un aspecto esencial a la hora de analizar el funcionamiento de este sector. De hecho, la situación geográfica de las estaciones de servicio resulta fundamental en la consideración de la compra de carburante por parte del consumidor. En este mismo sentido, la Comisión Nacional de la Energía (CNE) viene realizando desde mediados de 2007 estudios en entornos geográficos limitados (Vigo, Vitoria, etc.) sobre la evolución de precios por operadores y formas contractuales.

Este artículo ahonda en esta línea de investigación. Más concretamente, se centra en el análisis del efecto que tienen los factores locales de oferta en la fijación de los precios de los carburantes de automoción en las estaciones de servicio (EESS) españolas, cuestión que hasta ahora no ha sido estudiada en profundidad. Esto permitirá alcanzar un mayor nivel de conocimiento acerca del funcionamiento competitivo de este sector. Para ello, se utiliza una muestra de 529 estaciones de servicio representativas de toda la red española, para las cuales se han obtenido los precios de comercialización de gasolinas y gasóleos durante 39 semanas. Esta base de datos, además de recoger un conjunto de variables que miden los factores de localización que se pretenden estudiar, incorpora información acerca de la marca de abanderamiento y de los servicios ofrecidos en las estaciones de servicio. De esta forma, este trabajo utiliza una sólida base de datos representativa de la red de estaciones de servicio de todo un país, recogiendo, por primera vez en España una amplia información sobre las dimensiones verticales y horizontales de la competencia.

El artículo queda estructurado de la siguiente manera. En el apartado 2 se presenta una breve revisión de la literatura relacionada sobre competencia y fijación de precios en la distribución de carburantes de automoción. En el apartado 3, se especifica el modelo y se describe en detalle los efectos esperados que las variables que miden los factores de localización y de diferenciación pueden tener sobre los precios finales. En el 4, se presenta la muestra de estaciones de servicio que se utiliza para este estudio, el proceso de obtención de datos y la estadística descriptiva. En el apartado 5 , se justifica la metodología econométrica utilizada y se presentan los resultados. La discusión y las conclusiones cierran el trabajo.

${ }^{3}$ Como era de esperar, los principales operadores del sector discrepan de ésta y otras afirmaciones (http://www.aop.es/informes/informes_sector/comentarios_AOP_informe_CNC.pdf) 


\section{Literatura relacionada}

La literatura especializada en economía industrial ha analizado en detalle el sector de la distribución y comercialización de carburantes de automoción. Este sector se caracteriza por la presencia de grandes operadores integrados verticalmente que compiten con operadores menores y empresarios independientes en la comercialización de un bien básico de consumo masivo, poniendo en práctica distintas estrategias empresariales que permiten diferenciar horizontal y verticalmente unas estaciones de servicio de otras. Estas características peculiares han propiciado la realización de numerosos estudios académicos que se han centrado fundamentalmente en el análisis del comportamiento de los precios mayoristas y de comercialización de las gasolinas. Entre estos trabajos destacan por su relevancia, los que analizan la existencia de comportamientos anticompetitivos por parte de los operadores en distintos mercados de Norteamérica, utilizando varios modelos teóricos desarrollados a partir de la teoría de juegos, así como a partir de distintas aproximaciones empíricas que utilizan series temporales y datos de panel. Los trabajos más relevantes en este campo son los de Slade (1986, 1993), Shepard (1991), Borenstein (1991), Borenstein y Shepard (1996), Wang (2008), entre otros.

Desde una perspectiva similar, la atención se ha centrado también en el estudio del impacto de las relaciones contractuales que se establecen entre operadores y estaciones de servicio sobre los precios de comercialización (Shepard, 1993; Rey y Stiglitz, 1995; Hastings, 2004; Bello y Cavero, 2007). En general, se ha constatado que la existencia de diferencias en cuanto al poder de negociación de los operadores permite a estos imponer restricciones verticales aguas abajo para solucionar problemas de agencia y de doble marginalización, con resultados dispares en cuanto a su efecto en los precios finales (Slade, 1986; Shepard, 1993).

Desafortunadamente en España, los más de sesenta años de vigencia del monopolio de petróleos, el subsecuente sistema de regulación de precios máximos vigente para las gasolinas hasta septiembre de 1998, y las posteriores dificultades para obtener datos oficiales de precios a nivel de estaciones de servicios hasta el año 2006, ha hecho imposible replicar estudios similares de precios utilizando datos a nivel de operador o de estación de servicio. Por ello, la mayoría de los trabajos se han realizado, o bien utilizando datos agregados a nivel nacional y regional, o bien realizando análisis a nivel de estaciones de servicio pero de corte transversal. Entre estos, destacan los trabajos que desde la perspectiva de política de regulación y competencia se han centrado en el estudio de las características estructurales de la industria y sus efectos en el proceso de apertura y liberalización hacia la competencia (Correljè, 1990; Contín et al., 1999; 2001; Perdiguero y Borrel, 2007; Bello y Cavero, 2008). En general, se ha constatado la importancia de las barreras a la entrada existentes tanto a nivel de distribución mayorista como en la comercialización final. Por otra parte, también utilizando datos agregados a nivel de España, Contín-Pilart et al. (2008; 2009) han estudiado la existencia de asimetrías en la fijación de precios de gasolinas y gasóleos, rechazando su existencia para el mercado español. Por su parte, Perdiguero 
y Jiménez (2009) realizan un análisis de colusión de precios utilizando variaciones conjeturales para las Islas Canarias estimando un parámetro de conducta medio del conjunto de operadores que no permite rechazar al 5\% la existencia de colusión. Por último, utilizando datos a nivel de estaciones de servicio sobre precios, contratos y servicios ofrecidos pero de corte transversal, Bello y Cavero (2007) ponen en evidencia el impacto positivo de la marca de abanderamiento sobre los precios finales, y evidencian que la estrategia de separación vertical por medio de contratos en exclusiva se utiliza por los operadores para aumentar los precios y suavizar la competencia en el mercado final.

La riqueza de la base de datos con la que contamos en este trabajo nos permite hacer una aproximación novedosa al comportamiento de los precios de los carburantes de automoción en España, considerando que, además de las cotizaciones internacionales de las materias primas, la variabilidad de los precios de los carburantes es consecuencia de la existencia de diferenciación horizontal y vertical de las estaciones de servicio donde se comercializan los productos. Es decir, tal y como señala Hastings (2004), las estaciones de servicio se diferencian por su marca, su localización y por los servicios que ofrecen. En este sentido, si bien la calidad del producto y el nivel de servicios ofrecidos han demostrado ser determinantes de los precios de comercialización final (Borenstein, 1991; Shepard, 1991; Hastings, 2004; Hasting y Gilbert, 2005), los trabajos más recientes ponen especial énfasis en los factores de localización -distancias entre estaciones de servicio y configuración del mercado local en cuanto a número de competidores y sus marcas de abanderamiento- como los factores que tienen mayor poder explicativo de la variabilidad de los precios (Netz y Taylor, 2002; Barron et al., 2004; Hastings, 2004; Eckert y West, 2005; Clemenz y Gugler; 2006; Lewis, 2008; Pennerstofer, 2009).

En cuanto a los niveles de competencia local, Eckert y West (2005) consideran que la localización, la distancia a los competidores y la bandera bajo la cual operan estos, son los factores determinantes del modelo de competencia en el mercado de Vancouver. Los resultados de este trabajo ponen de manifiesto que la probabilidad de que una estación de servicio fije el "precio modal" o precios por encima de éste, depende principalmente de la distancia y la marca de la estación de servicio rival, además de otros factores de localización, aportando indicios de la no existencia de un modelo competitivo en aquel mercado. De forma similar, Clemenz y Gugler (2006) validan el modelo de competencia espacial para analizar la competencia en el mercado de distribución de carburantes de automoción en Austria y encuentran que el equilibrio de precios y la dispersión de los mismos se reducen conforme los competidores se sitúan más cerca. De esta forma, las empresas toman sus decisiones de localización buscando la máxima diferenciación espacial de sus productos para reducir los niveles de competencia (Netz y Taylor, 2002).

Con un enfoque similar, se señala la presencia de estaciones de servicio independientes como un factor mas dinamizador de la competencia, ya que la competencia aumenta considerablemente más con la presencia de éstas en el mercado relevante que con la presencia de estaciones de servicio abanderadas (Netz y Taylor, 2002) y 
consecuentemente los niveles de precios de comercialización se reducen (Hastings, 2004; Pennerstorfer, 2009). Por ello, en este trabajo se presta especial atención a la presencia de estaciones de servicio no abanderadas en los mercados locales. Esta evidencia abre la puerta a la introducción de competencia mediante la supervisión y regulación de los contratos entre operadores y estaciones de servicio según la configuración del mercado relevante.

\section{Especificación del Modelo}

Con el fin de conocer los factores explicativos de la fijación de precios de los carburantes de automoción a nivel de estación de servicio en España, vamos a estimar la siguiente ecuación:

$$
P_{i t}=f\left(\text { factores de localización }_{i}, \text { factores de diferenciación }_{i} \text {, } \text { cotización spot }_{i t}\right)
$$

donde $P_{i t}$, variable dependiente, es el precio semanal antes de impuestos de la gasolina y del gasóleo de automoción a nivel de estación de servicio. Los factores de localización $_{i}$, los factores de diferenciación ${ }_{i}$ y la cotización spot ${ }_{i t}$ de los carburantes, como proxy de su coste marginal de producción, son las variables explicativas. A continuación explicamos con detalle todas las variables explicativas utilizadas.

\subsection{Factores de localización}

\subsubsection{Estructura del Mercado y Distancias a Competidores}

La distancia que separa dos estaciones de servicio es un elemento fundamental para entender el comportamiento de los precios en los mercados locales. Eckert y West (2005) utilizan las distancias entre estaciones de servicio para estimar el modelo de competencia -perfecta, imperfecta-no colusiva, colusión-a partir de su efecto sobre la probabilidad de que una estación de servicio fije el "precio modal" del mercado o un precio superior a éste (precio de colusión). Si la competencia que se establece entre las estaciones de servicio es imperfecta-no colusiva, la mayor distancia de una estación de servicio respecto a sus competidoras se traducirá en mayores precios finales. Asimismo, en una situación de colusión, cuanto mayor sea la distancia que separa la estación de servicio que fija el precio de colusión de otra que no lo hace, mayor será la probabilidad de que haya un sostenimiento de precios elevados. En esta misma situación, según el teorema de Folk, cuanto menor sea la distancia entre estaciones de servicio que fijan el precio de colusión, menores son los incentivos a romper el acuerdo de precios, y por ello, mayor será la probabilidad de sostener los precios elevados. El que se dé esta situación de precios eleva- 
dos es más probable cuando la estación de servicio más cercana es de la misma marca. Por otra parte, si el modelo fuese de competencia no se esperaría un efecto de la distancia sobre los precios ya que este modelo se sostiene en la ausencia de costes de transporte e información por parte de los consumidores para elegir estaciones de servicio. En definitiva, es plausible que las estaciones de servicio no sean substitutos perfectos y que la localización y la marca de los competidores afecte a los precios finales de venta.

En nuestra investigación, y siguiendo la línea de otros trabajos (Eckert y West, 2005; Van Meerbeck, 2003), se hace especial hincapié en el efecto que la distancia a los competidores más cercanos y la estructura del mercado local -definido por el número y tipo de estaciones de servicio localizadas en un radio de dos kilómetros a una estación dada- tiene en los precios finales de venta. De esta forma, además de la variable de distancia al competidor más cercano (Distancia), se recoge el número de estaciones de servicio en un radio de dos kilómetros (Densidad) y la proporción de éstas que operan con la misma marca de abanderamiento (Densidad-Marca). Finalmente, por medio de una variable dicotómica medimos el impacto que sobre los precios de comercialización tiene la presencia de al menos una estación de servicio independiente en dicho mercado local (No Abanderada). Los coeficientes estimados de estas variables nos permitirán inferir las características más destacables del modelo de competencia que define al sector, como por ejemplo el efecto que sobre la competencia y los precios finales tiene la presencia de estaciones de servicio independientes en los mercados locales (Netz y Taylor, 2002; Hasting, 2004; Hastings y Gilbert, 2005; Clemenz y Gugler, 2006).

\subsubsection{Otros Factores de Localización}

El tipo de vía y el entorno donde operan las estaciones de servicio constituyen otros factores de la dimensión espacial de la competencia con efectos sobre los precios de comercialización de los carburantes de automoción. Por un lado, las autopistas de peaje en España presentan varias características peculiares que permiten presuponer precios superiores. En estas vías, a diferencia de lo que sucede en las carreteras nacionales o comarcales, los automovilistas tienen que hacer frente a un coste importante (tiempo y tasa de peaje) si abandonan la vía para repostar. Por otra parte, las concesiones de áreas de servicio en autopistas permiten la adquisición de varias zonas contiguas por parte de un único operador, hecho que se traduce en la existencia de situaciones de monopolio en estas vías cuando un mismo operador controla un largo trayecto de la vía.

Por otra parte, en las vías de circulación localizadas en centros urbanos, el menor coste de información y transporte al que hacen frente los consumidores para decidir dónde repostar como consecuencia de la mayor densidad de estaciones de servicio, hacen que, a priori y dependiendo de los niveles de concentración local, sean esperables unos precios más competitivos. 
Finalmente, en los polígonos industriales es frecuente encontrar hipermercados, que en general se caracterizan por ser muy agresivos en precios para atraer consumidores a sus centros comerciales, lo que puede generar situaciones de mayor competencia en estas zonas y con ello, la consecuente reducción de precios finales de comercialización de los carburantes de automoción.

En este trabajo, el tipo de vía donde operan las estaciones de servicio se recoge por medio de variables dicotómicas que toman valor uno cuando estas son autopistas con peaje (Autopista), carreteras nacionales (Carretera), vías en zonas residenciales (Residencial), vías en centros urbanos (CentroCiudad), o vías suburbanas en zonas industriales (Industrial), y cero en caso contrario.

\subsection{Factores de diferenciación}

\subsubsection{Marca de Abanderamiento}

La utilización de la marca como un elemento diferenciador entre estaciones de servicio es una estrategia que se ha generalizado en este sector. En general, se observa que la competencia se establece entre grandes operadores petroleros integrados verticalmente con larga trayectoria en los mercados nacionales compitiendo con marcas de operadores menores y con estaciones de servicio independientes. Los primeros se caracterizan por realizar un esfuerzo notable en diferenciación de sus estaciones de servicio por medio de promociones, publicidad y campañas de fidelización, mientras que, en el otro extremo, las estaciones de servicio no abanderadas centran sus esfuerzos en competir más intensamente en precios (Hastings, 2004; Bello y Cavero, 2008).

Esta generalización de las estrategias de marca en el sector ha propiciado su utilización como variable explicativa de los precios de comercialización de gasolinas y gasóleos en múltiples trabajos, ratificando en prácticamente todos ellos su efecto positivo sobre los precios finales (Shepard, 1991; Png y Reitman, 1995; Hastings, 2004; Bello y Cavero, 2008; Lewis, 2008; Hosken, McMillan y Taylor, 2008, entre otros).

En este trabajo, cada estación de servicio ha sido identificada por su marca de abanderamiento. Se distinguen, por un lado, las estaciones de servicio que operan con las marcas del Grupo Repsol-Ypf (Repsol), Cepsa (Cepsa) y BP (BP), ya que son los tres operadores con capacidad de refino en España, y por otro, se ha agrupado al resto de operadores petrolíferos que operan en este mercado y que no poseen capacidad de refino nacional con la variable "Otras Marcas". Por último, hemos identificado a las estaciones de servicio no abanderadas o independientes (No Abanderadas). Las evidencias existentes para el mercado español hacen esperar que los precios de comercialización de los operadores con mayor prestigio y reconocimiento a nivel local sean mayores que los precios de comercialización de los operadores independientes. 


\subsubsection{Servicios Ofrecidos}

Los servicios añadidos a la venta de carburantes que los usuarios pueden disfrutar en el momento de la compra de los carburantes (tiendas de conveniencia, servicios de hostelería y restauración, mecánica rápida, entre otros), pueden tener un importante impacto en los precios finales (Slade, 1998). Esto es así por que en general, los consumidores no realizan la compra basándose únicamente en los precios de la gasolina (Eckert y West, 2005). Así, si una gasolinera es autoservicio o no, si ofrece lavado o no, o si ofrece o no servicios de restauración o tiendas de conveniencia, puede ser un elemento determinante que oriente la elección de compra por parte de los consumidores. Asimismo, la presencia de servicios puede variar la percepción de calidad del establecimiento y con ello la predisposición a pagar por parte de los consumidores finales.

Por todo ello, en una situación de competencia imperfecta-no colusiva sería esperable un efecto positivo del nivel de servicios ofrecidos sobre los precios finales. Este mismo resultado podría observarse en una situación de colusión si los operadores fijasen precios de colusión diferenciados dependiendo de si se ofertan o no servicios en el punto de venta. Por último, en una situación de competencia perfecta no se espera sobreprecio como consecuencia de los servicios ofrecidos (Eckert y West, 2005).

Sin embargo, también es cierto que la utilización de estos servicios convierte a la gasolinera en un negocio más complejo y dinámico que el mero repostaje, donde los servicios, en muchos casos, constituyen un elemento esencial para la rentabilidad del establecimiento. Esta diversificación de productos en el punto de venta permitiría alcanzar economías de alcance en los establecimientos, lo que podría propiciar, dependiendo de la estrategia del establecimiento, una reducción de los precios de comercialización de los carburantes.

En este trabajo, hemos tenido en cuenta los servicios comúnmente considerados en la literatura especializada: Lavado Automático, Asistencia al Repostaje, Tienda de Conveniencia y Servicios de Reparación. Asimismo, hemos decidido agrupar los servicios ofrecidos en la gasolinera creando un índice acumulativo de servicios siguiendo los criterios de Bollen y Lennox (1991) y Bello y Cavero (2008), evitando así posibles problemas en la especificación del modelo como consecuencia de utilizar muchas variables dicotómicas.

\subsection{Cotización spot}

El precio spot de los carburantes de automoción es una proxy adecuada de su coste marginal de producción (Bacon, 1991). Si utilizáramos como referencia el precio spot del crudo haríamos depender el precio final de la gasolina (gasóleo), al menos en cierta medida, de la demanda de otros productos refinados dada la producción con- 
junta que caracteriza al refino ${ }^{4}$ (Borenstein et al., 1997). La inclusión de esta variable en nuestro análisis nos permitirá conocer en qué medida transmiten las estaciones de servicio los cambios que se producen en los precios spot a los precios finales antes de impuestos de los carburantes de automoción.

\section{Base de datos}

Las estaciones de servicio utilizadas en este estudio provienen de una muestra única representativa de la red española de estaciones de servicio. El procedimiento de recolección de datos se inició con la identificación de las estaciones de servicio de la red ${ }^{5}$, distinguiendo por marca de abanderamiento y por Comunidad Autónoma (CCAA), exceptuando las Islas Canarias. Posteriormente, se realizó un muestreo estratificado, proporcional a la participación de los distintos operadores en cada CCAA para lo cual se utilizaron los datos publicados en la Enciclopedia Española del Petróleo, Petroquímica y Gas (2007). De esta forma, la composición de la muestra quedó definida por un total de 529 estaciones de servicio en donde todas las provincias están representadas. Posteriormente, la empresa Catalist España seleccionó de forma aleatoria las estaciones de servicio concretas que componen la muestra. Para cada una de estas estaciones, se adquirió información referente a su localización, características del mercado dónde competían y los servicios que ofrecían en el año 2007 a la propia Catalist España.

Los precios de la gasolina de 95 octanos y del gasóleo A de automoción de las EESS de la muestra son los publicados por el Ministerio de Industria, Comercio y Turismo (http://geoportal.mityc.es/hidrocarburos/eess/), todos los lunes del período comprendido entre el 1 de febrero y el 30 de noviembre de $2007^{6}$. Se logró obtener la información necesaria para construir todas las variables que utilizamos en este trabajo para el $91,5 \%$ de las estaciones de servicio de la muestra, completando así un total de 484 gasolineras perfectamente identificadas por sus características y por los precios a los que comercializan los carburantes de automoción a lo largo de 39 semanas del año 2007. Los precios utilizados en las estimaciones son los precios de los carburantes de automoción antes de impuestos.

Nuestra muestra refleja adecuadamente el peso que tienen los distintos operadores petroleros en el sector de la distribución de carburantes de automoción a través de estaciones de servicio, ya que su participación en la muestra refleja adecuadamente su cuota de mercado ${ }^{7}$. En lo que respecta a los precios, nuestra muestra refleja

${ }^{4}$ Del crudo del petróleo se obtienen una amplia variedad de productos, como gasóleos, gasolinas, naftas, queroseno, etc.

${ }^{5}$ Directorio de Estaciones de Servicio de la Revista Estaciones de Servicio para el año 2007. Tecnipublicaciones España, S.L. Madrid.

${ }^{6}$ Según el artículo 4 del Real Decreto 6/2000 todas las estaciones de servicio de la red española están obligadas a remitir los precios de comercialización de hidrocarburos los lunes de cada semana.

${ }^{7}$ Las cuotas de mercado de los distintos operadores en el año 2006 fueron: 43,7\%, Repsol; 18,3, Cepsa; 7\%, BP; 17\%, Otras Marcas; y 14\%, No abanderadas, (Enciclopedia Nacional del Petróleo, Petroquímica y Gas, 2007). 
fielmente la evolución de los precios antes de impuestos en España. Así, el Gráfico 1 muestra cómo prácticamente coinciden los precios medios mensuales de nuestra muestra, tanto para la gasolina (Pga) como para el gasóleos (Pgl), con los estimados por el Ministerio de Industria (Pgamityc, caso de la gasolina; Pglmityc, caso del gasóleo ${ }^{8}$ ) para el período que nosotros analizamos. Por último, el rango de variación de precios entre CCAA (Cuadro 2) es muy similar el estimado por el citado Ministerio de Industria.

\section{GRÁFICO 1}

\section{COMPARATIVA DE LOS PRECIOS MENSUALES DE LOS CARBURANTES DE AUTOMOCIÓN ANTES DE IMPUESTOS (CENT€/LITRO)}

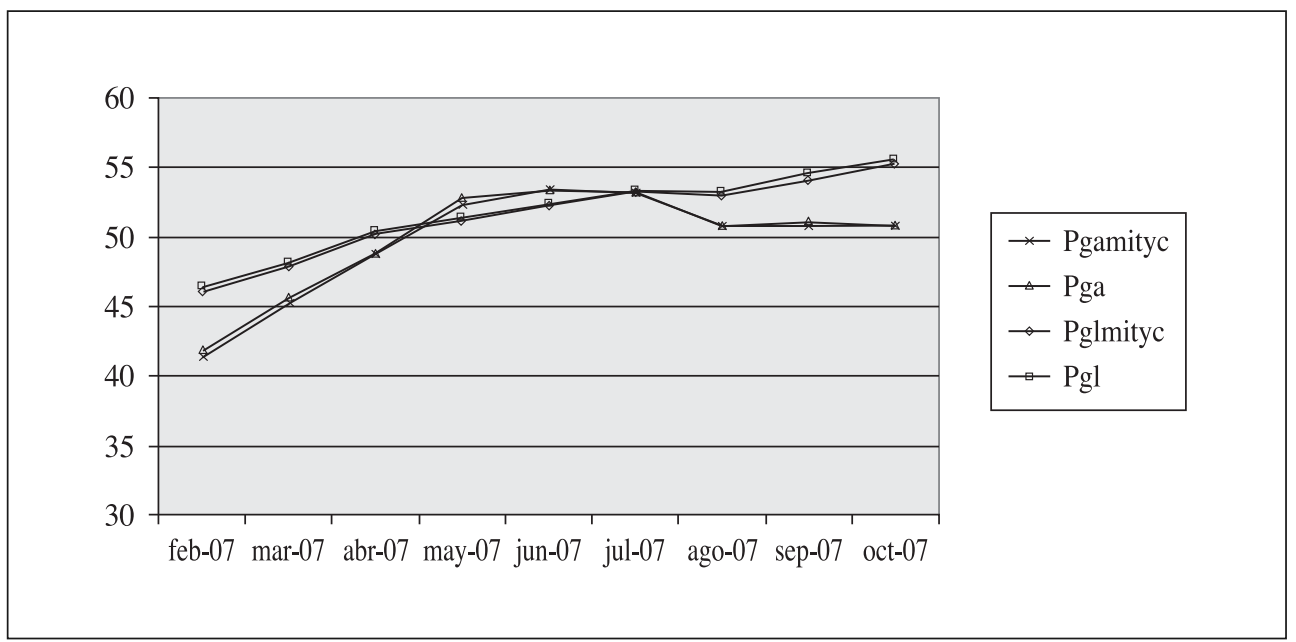

Fuente: Ministerio de Industria, Comercio y Turismo y muestra propia.

Analizando la estadística descriptiva acerca de los factores de localización que se presentan en el cuadro 1, se observa que, en media, las estaciones de servicio de la muestra se distancian de su competidor más cercano en 3,10 kilómetros, con una desviación típica de 4,10 kilómetros. La estación de servicio que opera de forma más aislada, lo hace a 32 kilómetros de distancia a su rival más próximo. Por otra parte, si observamos las características del mercado local, que se ha definido en un radio de 2 kilómetros alrededor de una estación de servicio dada, observamos que, en media, son aproximadamente dos estaciones de servicio las que compiten con cada gasolinera. Además, en el $23 \%$ de las estaciones de servicio de la muestra el mercado local de las mismas está constituido únicamente por estaciones de servicio de la misma marca. En esta misma área de influencia, en el 10\% de los casos existe la presencia de al menos una estación de servicio independiente.

${ }^{8}$ http://www.mityc.es/energia/petroleo/Paginas/Index.aspx. 


\section{CUADRO 1}

ESTADÍSTICA DESCRIPTIVA DE LA MUESTRA (484 ESTACIONES)

\begin{tabular}{|c|c|c|c|c|c|}
\hline & Media & $\begin{array}{l}\text { Desv. } \\
\text { Típica }\end{array}$ & Mín. & Máx. & $\mathbf{N}^{\circ}$ Obs. \\
\hline $\begin{array}{l}\text { Pit }_{\text {gasolina }} \text { - Precio de la gasolina } 95 \text { octanos } \\
\text { antes de impuestos. Céntimos de euro/li- } \\
\text { tro. }\end{array}$ & 49,92 & 3,77 & 36,48 & 59,07 & 18.954 \\
\hline $\begin{array}{l}\text { Pit }_{\text {gasoleo }}-\text { Precio del gasóleo A antes de im- } \\
\text { puestos. Céntimos de euro/litro. }\end{array}$ & 51,82 & 2,93 & 40,60 & 59,92 & 18.954 \\
\hline $\begin{array}{l}C_{\text {gasolina }}-\text { Cotización spot la gasolina-Platts } \\
\text { Rotterdam. Céntimos de euro/litro. }\end{array}$ & 38,68 & 3,43 & 30,27 & 44,04 & 18.954 \\
\hline $\begin{array}{l}C_{\text {gasoleo }} \text { - Cotización spot del gasóleo-Platts } \\
\text { Rotterdam. Céntimos de euro/litro. }\end{array}$ & 38,00 & 2,83 & 32,77 & 45,00 & 18.954 \\
\hline $\begin{array}{l}\text { Distancia en kilómetros a la estación de ser- } \\
\text { vicio más cercana. }\end{array}$ & 3,10 & 4,10 & 0,01 & 32,70 & 18.954 \\
\hline $\begin{array}{l}\text { Número de estaciones de servicio en un ra- } \\
\text { dio de dos kilómetros. }\end{array}$ & 1,92 & 2,34 & 0,00 & 9,00 & 18.954 \\
\hline $\begin{array}{l}\text { Porcentaje de estaciones de servicio para } \\
\text { las que sus mercados locales (dos kiló- } \\
\text { metros de radio) están constituidos úni- } \\
\text { camente por estaciones de servicio que } \\
\text { operan con la misma marca. }\end{array}$ & $23 \%$ & & 0 & $100 \%$ & 18.954 \\
\hline $\begin{array}{l}\text { Porcentaje de estaciones de servicio que en } \\
\text { un radio de dos kilómetros tienen al me- } \\
\text { nos una estación de servicio No-Aban- } \\
\text { derada. }\end{array}$ & $9,61 \%$ & & 0 & $100 \%$ & 18.954 \\
\hline $\begin{array}{l}\text { Porcentaje de estaciones de servicio loca- } \\
\text { lizadas en autopistas de peaje. }\end{array}$ & $2,27 \%$ & & 0 & $100 \%$ & 18.954 \\
\hline $\begin{array}{l}\text { Porcentaje de estaciones de servicio loca- } \\
\text { lizadas en carreteras nacionales, comar- } \\
\text { cales, autovías, etc. }\end{array}$ & $31,26 \%$ & & 0 & $100 \%$ & 18.954 \\
\hline $\begin{array}{l}\text { Porcentaje de estaciones de servicio loca- } \\
\text { lizadas en zonas residenciales. }\end{array}$ & $5,29 \%$ & & 0 & $100 \%$ & 18.954 \\
\hline $\begin{array}{l}\text { Porcentaje de estaciones de servicio loca- } \\
\text { lizadas en ciudades. }\end{array}$ & $27,78 \%$ & & 0 & $100 \%$ & 18.954 \\
\hline $\begin{array}{l}\text { Porcentaje de estaciones de servicio loca- } \\
\text { lizadas en polígonos industriales. }\end{array}$ & $33,08 \%$ & & 0 & $100 \%$ & 18.954 \\
\hline
\end{tabular}


CUADRO 1 (continuación)

ESTADÍSTICA DESCRIPTIVA DE LA MUESTRA (484 ESTACIONES)

\begin{tabular}{|c|c|c|c|c|c|}
\hline & Media & $\begin{array}{l}\text { Desv. } \\
\text { Típica }\end{array}$ & Mín. & Máx. & $\mathbf{N}^{\circ}$ Obs. \\
\hline $\begin{array}{l}\text { Proporción de estaciones de servicio que } \\
\text { operan con la marca Repsol*. }\end{array}$ & 0,46 & & 0,00 & 1,00 & 18.954 \\
\hline $\begin{array}{l}\text { Proporción de estaciones de servicio que } \\
\text { operan con la marca Cepsa }\end{array}$ & 0,18 & & 0,00 & 1,00 & 18.954 \\
\hline $\begin{array}{l}\text { Proporción de estaciones de servicio que } \\
\text { operan con la marca BP. }\end{array}$ & 0,07 & & 0,00 & 1,00 & 18.954 \\
\hline $\begin{array}{l}\text { Proporción de estaciones de servicio que } \\
\text { operan con Otras Marcas. }\end{array}$ & 0,18 & & 0,00 & 1,00 & 18.954 \\
\hline $\begin{array}{l}\text { Proporción de estaciones de servicio que } \\
\text { son No-Abanderadas. }\end{array}$ & 0,12 & & 0,00 & 1,00 & 18.954 \\
\hline Indice de Servicios ${ }^{* *}$. & 1,82 & 0,84 & 0,00 & 4,00 & 18.954 \\
\hline
\end{tabular}

Fuente: Ministerio de Industria, Comercio y Turismo; Platts; y muestra propia.

* Estaciones de Servicio del Grupo Repsol: Repsol, Campsa y Petronor.

** Índice de Servicios se distribuye entre 0 y 4 en función del número de servicios que se ofrece.

El 66\% de las estaciones de servicio de la muestra están instaladas en centros de ciudades, zonas residenciales e industriales. El 31\% de estaciones de servicio operan en carreteras y autovías nacionales y comarcales, mientras que el $3 \%$ de las estaciones de servicio de la muestra se localizan en autopistas con peaje. Por último, el nivel de servicios ofrecidos varía entre 0 y 4 , situándose la media cerca de dos.

En cuanto a los precios antes de impuestos, la estadística descriptiva que se presenta en el cuadro 2, muestra una importante dispersión de precios para el período analizado. El rango de precios es de 22,6 céntimos de euro/litro para la gasolina y de 19,32 céntimos de euro/litro para gasóleo A para todo el período y para toda la muestra. Sin embargo, esta información de precios exige un análisis descriptivo más pormenorizado, en el cual se recojan las variaciones de precios medios entre meses y entre regiones y dentro de las distintas regiones. En este sentido, tal y como se recoge en el cuadro 2, los rangos ante mencionados se explican en gran medida por el efecto temporal. El rango de medias mensuales para todas las estaciones de la muestra se sitúa en 11,6 y 8,57 céntimos de euro, para gasolinas y gasóleos, respectivamente. Sin embargo, las diferencias de precios medios antes de impuestos que existen entre las distintas regiones para todo el período son mucho menos acu- 
sadas, ya que el rango de medias es de 1,44 céntimos de euro para la gasolina de 95 octanos y de 1,56 céntimos de euro para el gasóleo $\mathrm{A}^{9}$.

CUADRO 2

ESTADÍSTICA DESCRIPTIVA DE LOS PRECIOS DE GASOLINAS Y GASÓLEOS ANTES DE IMPUESTOS (CENT€/LITRO)

\begin{tabular}{|l|c|c|c|}
\hline & $\begin{array}{c}\text { Total } \\
\text { de Observaciones }\end{array}$ & $\begin{array}{c}\text { Medias } \\
\text { Mensuales }\end{array}$ & $\begin{array}{c}\text { Medias } \\
\text { Regionales }\end{array}$ \\
\hline GASOLINA & 49,91 & 49,91 & 49,91 \\
\hline Media & 3,76 & 3,84 & 0,345 \\
\hline Desviación Stándar & 59,07 & 53,40 & 49,35 \\
\hline Máximo & 36,48 & 41,80 & 50,79 \\
\hline Mínimo & 18.954 & 9 & 16 \\
\hline Número de Observaciones & & & \\
\hline GASÓLEO & 51,81 & 51,81 & 51,81 \\
\hline Media & 2,93 & 2,95 & 0,42 \\
\hline Desviación Stándar & 59,92 & 55,05 & 52,62 \\
\hline Máximo & 40,6 & 46,48 & 51,06 \\
\hline Mínimo & 18.954 & 9 & 16 \\
\hline Número de Observaciones & & & \\
\hline
\end{tabular}

Fuente: Muestra propia.

No obstante, la muestra refleja que, en general, la dispersión de precios que existen dentro de cada CCAA y en un mismo momento del tiempo es notablemente superior a la existente entre regiones (cuadro 3$)^{10}$.

${ }^{9}$ Estos rangos son similares a los rangos mensuales de precios entre CCAA que el Ministerio de Industria estima para 2007.

${ }^{10}$ Asimismo, la diferencia de precios antes de impuestos entre la estación de servicio más cara y la más barata para el conjunto de España, estimada a partir de los datos de nuestra muestra y en un mismo momento del tiempo, rondaría en media los 14,18 céntimos de euro por litro para la gasolina y los 11,5 para el gasóleo. 


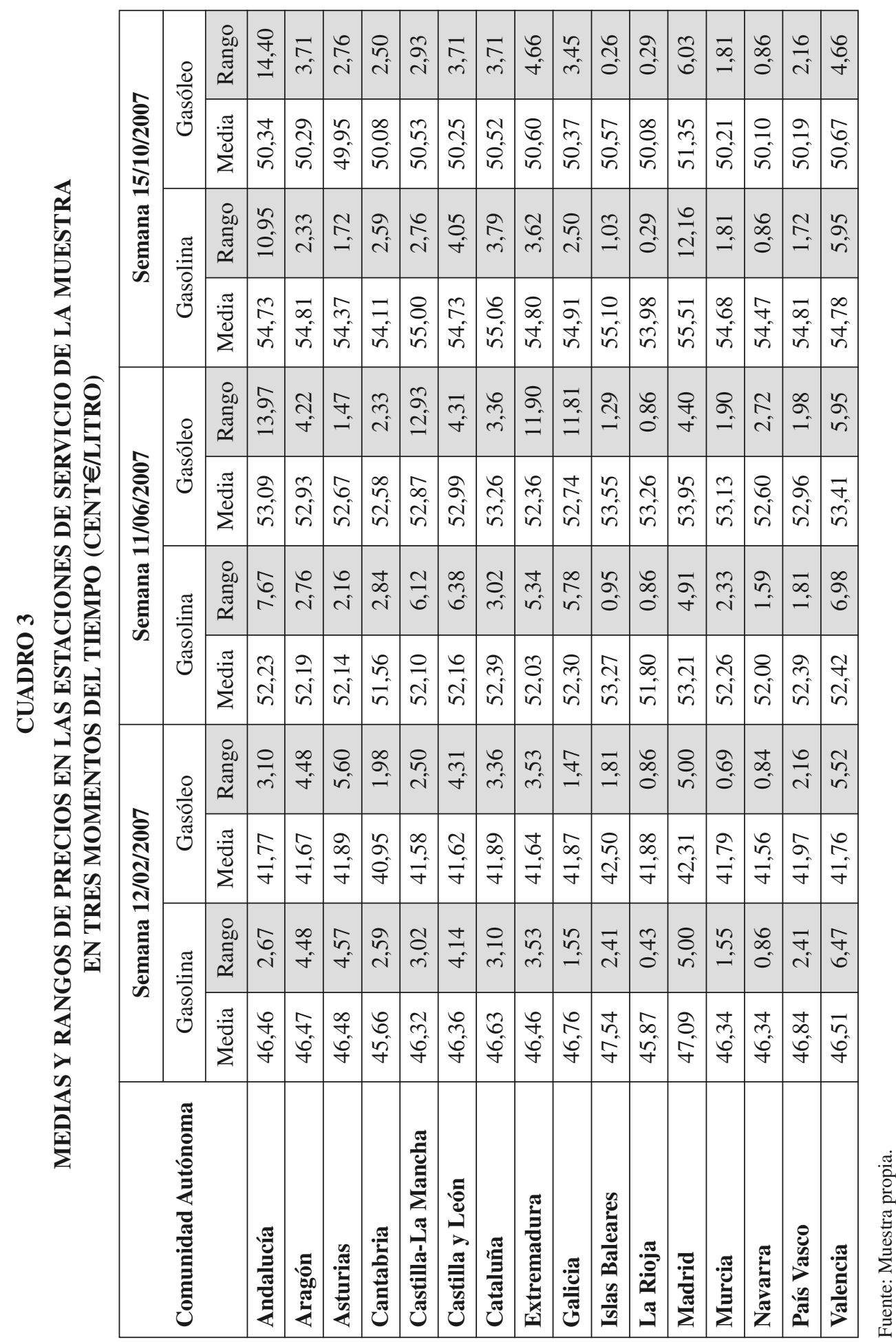




\section{Metodología econométrica y resultados}

Estimamos los parámetros de la ecuación (1) a partir del siguiente modelo (regresión agrupada o pooled):

$$
P_{i t}=\phi+X_{i t} \beta+\mu_{i t}
$$

donde

$\phi \quad$ es el término constante;

$X_{i t}$ es el vector de las variables explicativas y

$\mu_{i t}$ es el término de error aleatorio.

Es decir, estimamos el precio semanal antes de impuestos a nivel de estación de servicio, $P_{i t}$, tanto para la gasolina como para el gasóleo, en función de factores de localización, diferenciación y de la cotización spot de los carburantes de automoción. Realizamos la estimación por MCO con errores estándares de Newey-West robustos a la autocorrelación y heteroscedasticidad.

Las estaciones de servicio fijan los precios antes de impuestos en función del coste de los inputs, fundamentalmente en función del coste de los carburantes de automoción, y en función de los factores de localización que les afectan, tanto si estamos hablando de estaciones de servicio que operan baja la marca de un operador al por mayor ${ }^{11}$ como de estaciones de servicio no abanderadas.

Los cuadros 4 y 5 presentan las estimaciones. Más concretamente, la primera columna de cada tipo de carburante de automoción presenta una versión reducida de la ecuación (1), en la que incluimos como variables explicativas los factores de localización y la cotización spot. En la segunda columna mostramos los resultados incluyendo todas las variables explicativas.

Con respecto a los factores de localización, tanto para la gasolina como para el gasóleo, obtenemos los mismos resultados. Así, la variable distancia no es estadísticamente significativa, lo que sugiere que el hecho de que la estación más próxima a una dada esté más o menos alejada no tiene ningún efecto estadísticamente relevante sobre los precios fijados por aquella. Por el contrario, sí es estadísticamente significativa la variable densidad, lo que nos indica que cuanto mayor es el número de estaciones de servicio que una determinada estación tiene en un radio de dos kilómetros mayores son los precios que fija para los carburantes de automoción. Asimismo, el porcentaje de estaciones de la misma marca que una dada

${ }^{11}$ Esta situación es independiente del tipo de contrato que se establezca entre el operador y la estación de servicio que lleva su marca. En todos estos casos, los operadores además de mantener un fuerte control sobre la fijación de precios a nivel de estación de servicio (CNC, 2009) siguen una estrategia de marca para el conjunto de estaciones de servicios de su red. 
en un ratio de dos kilómetros, una vez controlados los efectos de diferenciación, no tiene un efecto significativo sobre los precios de aquella; pero sí el que haya al menos una estación de servicios no abanderada en ese mismo radio, variable que, como esperábamos, tiene un efecto negativo y significativo. Por lo tanto, solamente la presencia de estaciones no abanderadas parece que intensifica la competencia en el ámbito local en el sector petrolero español, y no así el incremento del número de estaciones de servicio per se, que incluso se podría traducir en una aumento de precios.

Como era de esperar, el hecho de que una estación de servicio se encuentre en una autopista tiene un efecto positivo y significativo sobre los precios, especialmente en el caso del gasóleo. El que una estación de servicio esté en un tipo de vía u otro no tiene ningún efecto estadísticamente significativo sobre el precio de la gasolina, pero sí en el caso del gasóleo donde observamos que las estaciones ubicadas en zonas industriales ofrecen precios más bajos que el resto. Las gasolineras presentes en esta zonas podrían fijar precios competitivos para el gasóleo con el objetivo de atraer a los camiones que llegan a cargar/descargar productos en estas zonas. Los aumentos en los volúmenes de ventas totales (litros por estación) que pueden acarrear este tipo de estrategias de precios podrían compensar los precios relativamente bajos de venta finales.

Con respecto a los factores de diferenciación, observamos diferencias significativas entre la gasolina y el gasóleo. Así, vemos como para la gasolina las estaciones de servicio con marcas conocidas y diferentes a las refineras, las no abanderadas y las de Repsol son las que ofrecen los precios más baratos, siendo las estaciones de servicio de Cepsa y BP las que tienen mayores precios. Para el gasóleo, vemos cómo las diferencias de precios entre distintos tipos de estaciones de servicio son relativamente más acusadas que en el caso de la gasolina, y cómo las estaciones de servicio que operan bajo las marcas de las empresas refineras establecidas en España cargan sistemáticamente mayores precios que las estaciones que operan bajo otras marcas y que las no abanderadas. Las variables de servicios presenta el signo esperado, ya que a mayores servicios, mayor precio.

Por último, tanto para la gasolina como para el gasóleo rechazamos la hipótesis nula de que el coeficiente del precios spot es igual a uno $(\mathrm{F}=15.577,46$, p-valor $=0,000$; $\mathrm{F}=146,79$, $\mathrm{p}$-valor $=0,000$; respectivamente), lo que sugiere que durante nuestro período de análisis los cambios que se han producido en el precio spot de los carburantes de automoción no se han transmitido en su totalidad al precio final antes de impuestos. 


\section{CUADRO 4 \\ RESULTADOS DE LA ESTIMACIÓN \\ PARA LA GASOLINA SIN PLOMO DE 95 OCTANOS}

\begin{tabular}{|c|c|c|c|c|c|c|}
\hline Variable & $\begin{array}{l}\text { Coeficiente } \\
\text { Estimado }\end{array}$ & $\begin{array}{c}\text { Newey-West } \\
\text { Errores Estándar }\end{array}$ & p-valor & $\begin{array}{l}\text { Coeficiente } \\
\text { Estimado }\end{array}$ & $\begin{array}{c}\text { Newey-West } \\
\text { Errores Estándar }\end{array}$ & p-valor \\
\hline Distancia & 0,0115 & 0,0120 & 0,342 & 0,0055 & 0,0117 & 0,638 \\
\hline Densidad & 0,0623 & 0,0213 & 0,004 & 0,0683 & 0,0213 & 0,001 \\
\hline Densidad-Marca & 0,0010 & 0,0012 & 0,406 & 0,0007 & 0,0012 & 0,546 \\
\hline No-Abanderada & $-0,4149$ & 0,1260 & 0,001 & $-0,3449$ & 0,1254 & 0,006 \\
\hline Autopista & 0,5703 & 0,2512 & 0,023 & 0,4329 & 0,2502 & 0,084 \\
\hline Carreteras & 0,0700 & 0,1078 & 0,516 & 0,1681 & 0,1128 & 0,136 \\
\hline Residencial & 0,0764 & 0,2166 & 0,724 & 0,1278 & 0,2156 & 0,553 \\
\hline Centro Ciudad & 0,0874 & 0,1091 & 0,423 & 0,0838 & 0,1128 & 0,457 \\
\hline Repsol & & & & $-0,0398$ & 0,1432 & 0,781 \\
\hline Cepsa & & & & 0,2982 & 0,1456 & 0,041 \\
\hline$B P$ & & & & 0,3729 & 0,1754 & 0,033 \\
\hline Otras & & & & $-0,2548$ & 0,1713 & 0,137 \\
\hline Servicios & & & & 0,1054 & 0,0470 & 0,025 \\
\hline Cotización spot & 0,9018 & 0,0073 & 0,000 & 0,9018 & 0,0072 & 0,000 \\
\hline Constante & 14,876 & 0,3034 & 0,000 & 14,629 & 0,3306 & 0,000 \\
\hline $\mathbf{F}$ & $1.766,02^{*}$ & & & $1.206,74^{*}$ & & \\
\hline R-sq & 0,6784 & & & 0,6814 & & \\
\hline N. Obs. & 18.954 & & & 18.954 & & \\
\hline
\end{tabular}

$* \mathrm{P}<0,001$. 


\section{CUADRO 5}

\section{RESULTADOS DE LA ESTIMACIÓN PARA EL GASÓLEO A DE AUTOMOCIÓN}

\begin{tabular}{|c|c|c|c|c|c|c|}
\hline Variable & $\begin{array}{l}\text { Coeficiente } \\
\text { Estimado }\end{array}$ & $\begin{array}{c}\text { Newey-West } \\
\text { Errores Estándar }\end{array}$ & p-valor & $\begin{array}{c}\text { Coeficiente } \\
\text { Estimado }\end{array}$ & $\begin{array}{l}\text { Newey-West } \\
\text { Errores Estándar }\end{array}$ & p-valor \\
\hline Distancia & 0,0120 & 0,0057 & 0,035 & 0,0050 & 0,0051 & 0,330 \\
\hline Densidad & 0,0622 & 0,01148 & 0,000 & 0,0665 & 0,0112 & 0,000 \\
\hline Densidad-Marca & 0,0017 & 0,0005 & 0,003 & 0,0003 & 0,0005 & 0,564 \\
\hline No-Abanderada & $-0,476$ & 0,0712 & 0,000 & $-0,4240$ & 0,0686 & 0,000 \\
\hline Autopista & 0,6483 & 0,1316 & 0,000 & 0,5226 & 0,1239 & 0,000 \\
\hline Carreteras & 0,1102 & 0,0535 & 0,039 & 0,1503 & 0,0547 & 0,006 \\
\hline Residencial & 0,1969 & 0,1036 & 0,057 & 0,2192 & 0,1028 & 0,033 \\
\hline Centro Ciudad & 0,2135 & 0,0564 & 0,000 & 0,1775 & 0,0584 & 0,002 \\
\hline Repsol & & & & 0,3009 & 0,0746 & 0,000 \\
\hline Cepsa & & & & 0,4159 & 0,0766 & 0,000 \\
\hline$B P$ & & & & 0,3114 & 0,0909 & 0,001 \\
\hline Otras & & & & $-0,0702$ & 0,0957 & 0,463 \\
\hline Servicios & & & & 0,0684 & 0,0223 & 0,002 \\
\hline Cotización spot & 0,925 & 0,0062 & 0,000 & 0,9259 & 0,0061 & 0,000 \\
\hline Constante & 16,41 & 0,2435 & 0,000 & 16,09 & 0,2564 & 0,000 \\
\hline $\mathbf{F}$ & $2.495,95^{*}$ & & & $1.720,20^{*}$ & & \\
\hline R-sq & 0,8071 & & & 0,8108 & & \\
\hline N. Obs. & 18.954 & & & 18.954 & & \\
\hline
\end{tabular}

* $\mathrm{P}<0,001$. 


\section{Discusión y Conclusiones}

Este trabajo analiza el efecto que los factores de localización tienen en las políticas de fijación de precios de los carburantes de automoción a nivel de estaciones de servicio en España. Para ello, se ha realizado un esfuerzo notable para construir una base de datos propia constituida por 529 estaciones de servicio, que representan la estructura competitiva del mercado español, y para las cuales se cuenta con la información de los precios fijados antes de impuestos durante 39 semanas en el año 2007, tanto para gasolinas como para gasóleos. Además, cada estación de servicio de la muestra es identificada con sus características de competencia local, es decir, distancias a competidores más cercanos, cantidad y marca de competidores locales y el tipo de vía donde se localiza, además de su marca de abanderamiento y de los servicios que ofrece.

Como principales conclusiones del trabajo destacamos las siguientes:

- En primer lugar, la información sobre precios agregados de comercialización antes de impuestos para gasolinas y gasóleos pone de manifiesto que, en efecto, y coincidiendo con la información facilitada por el Ministerio de Industria, Turismo y Comercio, las diferencias de precios que existen entre las distintas CCAA españolas no son muy acusadas. Sin embargo, si analizamos la dispersión de precios que existe dentro de cada región y en un mismo momento del tiempo, comprobamos que las diferencias de precios son notablemente más destacadas, y en algunos casos suponen más del $25 \%$ de los precios medios en una CCAA y en una semana dada. Este resultado desmitifica la creencia de que los precios de comercialización en este sector presentan muy bajos niveles de variación y justifica el estudio de los posibles motivos que explican esta variabilidad.

- En segundo lugar, las estimaciones de precios de gasolina y gasóleo ponen de manifiesto que los factores locales tienen, de forma significativa, capacidad explicativa sobre su variabilidad. En este sentido, el efecto de la densidad de estaciones de servicio en los mercados locales donde éstas operan, sugiere la existencia de imperfecciones en la competencia. Así, hemos observado que la existencia de un mayor número de estaciones de servicio en un radio de dos kilómetros alrededor de una determinada estación tiene un efecto positivo y significativo sobre los precios finales fijados por esta última. Esto sucede, además, independientemente de la marca con la que operan las estaciones de servicio más cercanas, ya que la presencia de estaciones de servicio de la misma marca en este mercado no tiene un efecto significativo. Este resultado tiene implicaciones para la política de la competencia, en tanto que desde la CNC y desde la CNE, y en reiteradas ocasiones, se ha señalado que la apertura de gasolineras como el elemento sustancial para introducir mayor competencia en el sector. Sin embargo, nuestros resultados ponen de manifiesto que la promoción de nuevas aperturas de estaciones de servicio en los mercados locales no garantiza per se que los niveles de competencia vayan a aumentar. 
Además, hemos observado que cuando en el mercado local donde opera una estación de servicio existe al menos una gasolinera independiente o no abanderada, los precios finales de comercialización, tanto en gasolinas como en gasóleos, se reducen de forma importante. Este resultado se alinea con los obtenidos en otros estudios nacionales y extranjeros en los que se confirma que la existencia de estaciones de servicio no abanderadas es un elemento dinamizador de la competencia y de la reducción de precios. Así, los esfuerzos para introducir mayores niveles de competencia en los mercados locales deberían centrarse en promover la presencia de estaciones de servicio de este tipo, bien por medio de nuevas aperturas, bien facilitando los cambios contractuales. Este tipo de medidas tendrían un mayor efecto en las autopistas de peaje, donde se observan de forma sostenida y sistemática niveles de precios superiores, y en las que se constata la ausencia de estaciones no abanderadas.

- Por último, nuestros resultados no permiten identificar diferencias en la fijación de precios antes de impuestos entre gasolinas y gasóleos en función de los parámetros de localización, ya que los coeficientes estimados son muy similares en magnitud y significatividad estadística. Sin embargo, las estimaciones que recogen el impacto de la marca sobre los precios finales, ponen de manifiesto la existencia de diferencias en las estrategias de precios por productos entre los operadores que cuentan con capacidad de refino en España. Más concretamente nuestros resultados sugieren que Repsol, al contrario que Cepsa y BP, estaría llevando a cabo una política de fijación de precios diferente en el mercado de gasolinas que en el de gasóleos, siendo más agresiva en el primer caso al no diferenciarse sus precios de los fijados por estaciones no abanderadas y con marcas reconocidas que no cuentan con capacidad de refino instalada en España. A su vez, esto determina que en el mercado de la gasolina se observen diferencias menos acusadas entre los distintos operadores con las estaciones de servicio independientes, sugiriendo que el nivel de competencia en este mercado es mayor que en el de gasóleos.

Estas evidencias revelan la necesidad de estudiar en futuras investigaciones las posibles causas que estén detrás de las diferencias observadas en las estrategias de fijación de precios de los operadores según el producto (gasolina o gasóleo), máxime teniendo en cuenta la notable importancia del consumo de gasóleo en España.

\section{Referencias Bibliográficas}

[1] BACON, R.W (1991): Rockets and feathers: the asymmetric speed of adjustment of UK retail gasoline price to cost changes, Energy Economics, 13, pp. 211-218.

[2] BARRON, J., TAYLOR, B. y UMBECK, J. (2004): Number of sellers, average prices, and price dispersion, International Journal of Industrial Organization, 22 (8-9), pp. 1041-1066. 
[3] BELLO, A. y CAVERO, S. (2007): Competencia estratégica en la distribución minorista de carburantes y combustibles de automoción en España, Revista de Economía Aplicada, 45, pp. 125-154.

[4] BELLO, A. y CAVERO, S. (2008): The Spanish retail gasoline market. New patterns of competition since the liberalization of the industry", Energy Policy, 36, pp. 612-626.

[5] BOLLEN, K. y LEENOX, R. (1991): Conventional wisdomon measurement: A structural equation perspective, Psychological Bulletin, 110(2), pp. 305-314.

[6] BORENSTEIN, S. (1985): Price discrimination in free-entry markets, Rand Journal of Economics, 16, pp. 380-397.

[7] BORENSTEIN, S. (1991): Selling costs and switching costs: Explaining retail gasoline margins", Rand Journal of Economics, 22, pp. 345-369.

[8] BORENSTEIN, S. y SHEPARD, A. (1996): Dynamic pricing in retail gasoline markets, Rand Journal of Economics, 27, pp. 429-451.

[9] BORENSTEIN, S.; CAMERON, C. y GILBERT, R. (1997): Do gasoline prices respond asymmetrically to crude oil prices?, Quarterly Journal of Economics, 112, pp. 305-339.

[10] CLEMENZ, G. y GUGLER, K. (2006): Locational choice and price competition: some empirical results for the Austrian retail gasoline market, Empirical Economics, 31, pp. 291-312.

[11] CNC (2009): Informe sobre la competencia en el sector de carburantes de automoción". Madrid. (http://www.cncompetencia.es/Inicio/Promocion/Informes/tabid/166/Default.aspx)

[12] CONTÍN, I.; CORRELJÉ, A. y HUERTA, E. (1999): The Spanish gasoline market: From ceiling regulation to open market pricing. The Energy Journal, 20(4), pp. 1-15.

[13] CONTÍN, I.; CORRELJÉ, A. y HUERTA, E. (2001): The Spanish distribution system for oil products: an obstacle to competition. Energy Policy, 29, pp. 885-895.

[14] CONTÍN-PILART, I.; CORRELJÉ, A. y PALACIOS, B. (2008): Asimetría de precios y evolución de márgenes comerciales en el mercado español del gasóleo de automoción, Hacienda Pública Española, 185 (2), pp. 9-37.

[15] CONTÍN-PILART, I.; CORRELJÉ, A. y PALACIOS, B. (2009): Competition, regulation and pricing behaviour in the Spanish retail gasoline market, Energy Policy, 37 (1), pp. 219-228.

[16] CORRELJÉ, A. (1990): The Spanish oil sector: From state intervention to free market, Energy Policy,18, pp. 747-755.

[17] ENCICLOPEDIA NACIONAL DEL PETRÓLEO, PETROQUÍMICA Y GAS (2007): Sede Técnica S.A, Madrid, España.

[18] ECKERT, A. y WEST, D. (2005): Price uniformity and competition in a retail gasoline market, Journal of Economic Behavior and Organization, 56, pp. 219-237.

[19] GARCIA DE COCA, J.A. (2004): Liberalización y competencia en el sector del petróleo. Balance 1998-2003, en Gaspar Ariño (Dir.), Privatizaciones y liberalizaciones en España: Balance y resultados (1996-2003), Tomo II, Granada, Comares Editorial, pp. 295-386.

[20] HASTINGS, J. (2004): Vertical relationships and competition in retail gasoline markets: Empirical evidence from contract changes in Southern California, The American Economics Review, 94 (1), pp. 317-328. 
[21] HASTINGS, J. y GILBERT, R. (2005): Market power, vertical integration and the wholesale price of gasoline, Journal of Industrial Economics, 53 (4), pp. 469-492.

[22] HOSKEN, D.; MCMILLAN, R. y TAYLOR, C. (2008): Retail gasoline pricing: What do we know?, International Journal of Industrial Organization, 26(6), pp. 1425-1436.

[23] LEWIS, M. (2008): Price dispertion and competition with differentiated sellers, Journal of Industrial Economics, 3, pp. 654-678.

[24] NETZ, S. y TAYLOR, B. (2002): Maximum or minimum diferentiation? Location patterns of retail outlets, The Review of Economics and Estatistics, 84 (1), pp. 162-175.

[25] PENNERSTORFER, D. (2009): Spatial price competition in retail gasoline markets: evidence from Austria, Annals of Regional Science, 43, pp. 133-158.

[26] PERDIGUERO, J. y BORREL, J. R. (2007): La difícil conducción de la competencia por el sector de gasolinas en España, Economía Industrial, 365, pp. 113-125.

[27] PERDIGUERO, J. y JIMÉNEZ, J. L. (2009): ¿Competencia o colusión en el mercado de gasolina? Una aproximación a través del parámetro de conducta, Revista de Economía Aplicada, 17 (50), pp. 27-45

[28] PNG, I. y REITMAN, D. (1995): Service time competition, Rand Journal of Economics, 25, pp. 619-634.

[29] REY, P. y STIGLITZ, J. (1995): The role of exclusive territories in producers' competition", Rand Journal of Economics, 26, pp. 431-451.

[30] SLADE, M. E. (1986): Exogeneity tests of market boundaries applied to petroleum products, Journal of Industrial Economics, 34 (3), pp. 291-303.

[31] SLADE, M. E. (1987): Interfirm rivalry in a repeated game: An empirical test of tacit collusion, Journal of Industrial Economics, 35, pp. 499-516.

[32] SLADE, M. E. (1993): Strategic motives for vertical separation: An empirical exploration, Journal of Law, Economics, and Organization, 14 (1), pp. 84-113.

[33] SLADE, M. E. (1998): Strategic motives for vertical separation: Evidence from retail gasoline markets, Journal of Law, Economics, and Organization, 14 (1), pp. 84-113.

[34] SHEPARD, A. (1991): Price discrimination and retail configuration, Journal of Political Economy, 99, pp. 30-54.

[35] SHEPARD, A. (1993): Contractual form, retail price, and asset characteristics in gasoline retailing, Rand Journal of Economics, 24, pp. 58-77.

[36] VAN MEERBECK, W. (2003): "Competition and local market conditions on the Belgian retail gasoline market”, De Economist, 151 (4), pp. 369-388.

[37] WANG, Z. (2008): Collusive communication and pricing coordination in a retail gasoline market, Review of Industrial Organization, 32, pp. 35-52. 
